\title{
Fluorine-18 fluorodeoxyglucose positron emission tomography imaging of T-lymphoblastic lymphoma patients
}

\author{
JONG HOON PARK ${ }^{*}$, KISOO PAHK ${ }^{2 *}$, SUNGEUN KIM ${ }^{2,3}$, SANG MOO LIM ${ }^{3}$, GI JEONG CHEON SH, $^{3,4}$ \\ YEON HEE PARK ${ }^{5,6}$, SEUNG-SOOK $\mathrm{LEE}^{7}$ and JAE GOL CHOE ${ }^{2}$
}

\begin{abstract}
${ }^{1}$ Musculoskeletal Oncology Clinic; ${ }^{2}$ Department of Nuclear Medicine, Korea University Medical Center Anam Hospital, Seoul 136-705; ${ }^{3}$ Department of Nuclear Medicine, Korean Institute of Radiological and Medical Sciences, Seoul 139-706; ${ }^{4}$ Department of Nuclear Medicine, Seoul National University Hospital, Seoul 110-744; ${ }^{5}$ Division of Hematology and Oncology, Department of Medicine, Samsung Medical Center, Seoul 06351; Departments of ${ }^{6}$ Internal Medicine and ${ }^{7}$ Pathology, Korean Institute of Radiological and Medical Sciences, Seoul 139-706, Republic of Korea
\end{abstract}

Received April 18, 2015; Accepted June 10, 2016

DOI: $10.3892 / \mathrm{ol} .2016 .4806$

\begin{abstract}
The purpose of the present study was to evaluate the fluorine-18 fluorodeoxyglucose positron emission tomography (FDG-PET) findings in patients with T-lymphoblastic lymphoma (T-LBL). In total, 9 patients with histopathologically confirmed T-LBL were included in the study. Bone marrow (BM) involvement and leukemic transformation (LT) were evaluated through iliac crest marrow biopsy and peripheral blood blast count. FDG-PET scans were performed at the initial pre-treatment point. Two experienced nuclear medicine physicians evaluated the FDG-PET images by visual analysis and using the maximum standardized uptake values (SUVmax) of the malignant lesions. Overall, 8 out 9 patients presented with BM involvement; 7 showed LT, while 1 showed BM involvement without LT. All involved T-LBL lesions were FDG-avid with variable uptake. The mean SUVmax was 6.4 \pm 3.3 . T-LBL patients with BM involvement showed diffuse or nodular marrow uptake. In addition, all the patients with LT showed diffuse marrow FDG activity. However, the patient with BM involvement but no LT showed nodular FDG uptake in the marrow. In conclusion, the present study indicates that it is possible to use FDG-PET for the evaluation of the disease extent of T-LBL. Furthermore, the imaging technique could provide a diagnostic clue for determining BM involvement or LT.
\end{abstract}

Correspondence to: Professor Sungeun Kim, Department of Nuclear Medicine, Korea University Medical Center Anam Hospital, 126-1 Anam-Dong 5-Ga, Seoul 136-705, Republic of Korea

E-mail: seiong@korea.ac.kr

Professor Sang Moo Lim, Department of Nuclear Medicine, Korean Institute of Radiological and Medical Sciences, 75 Nowongil, Seoul 139-706, Republic of Korea

E-mail: smlim328@kcch.re.kr

*Contributed equally

Key words: lymphoma, T-lymphoblastic lymphoma, fluorodeoxyglucose-positron emission tomography, positron emission tomography

\section{Introduction}

T-Lymphoblastic lymphoma (T-LBL) is a rare and aggressive subtype of adult non-Hodgkin's lymphoma (NHL) (1). The unique clinical and biological characteristics of T-LBL, including a male predominance and a high incidence of mediastinal tumors, differ from those of diffuse large B-lymphoblastic lymphoma, which is the most common type of NHL (1). In addition, T-LBL has a poor long-term survival rate with standard cyclophosphamide, doxorubicin, vincristine and prednisolone-like chemotherapy (2).

Fluorine-18 fluorodeoxyglucose positron emission tomography (FDG-PET) has been widely used for the evaluation of lymphoma, including NHL (3). With FDG-PET, it is possible to assess the lymphoma burden in the entire body, as FDG-PET is a more sensitive and specific diagnostic tool compared with other imaging modalities, such as computed tomography (3). Malignant and aggressive lymphomas typically exhibit a higher FDG uptake on FDG-PET scans (3). In addition, certain studies have reported the FDG-PET findings of lymphoma subtypes (4). However, only a few studies have described the FDG-PET findings in T-LBL patients $(4,5)$.

The aim of the present study was to evaluate FDG-PET images in patients with a specific subtype of lymphoma, T-LBL.

\section{Patients and methods}

Patients. In total, 9 patients ( 8 men and 1 woman; mean age, $30 \pm 8.3$ years) with initially histopathologically confirmed T-LBL at the Korean Institute of Radiological and Medical Sciences, Seoul, Republic of Korea, between January 2000 and December 2005, were retrospectively enrolled in the present study. The present study was approved by the Ethics Committee of the Korean Institute of Radiological and Medical Sciences (Institutional Review Board no. K-1507-002-026).

Histopathological diagnosis. Specimens obtained by lymph node or tumor biopsy were assessed by histological or cytological examination. Specimens were embedded with paraffin 
Table I. Characteristics of the 9 T-LBL patients.

\begin{tabular}{|c|c|c|c|c|c|c|c|c|c|c|}
\hline \multirow{2}{*}{$\begin{array}{l}\text { Case } \\
\text { no. }\end{array}$} & \multirow[b]{2}{*}{ Gender/age } & \multirow{2}{*}{$\begin{array}{l}\text { Histological } \\
\text { type }\end{array}$} & \multirow{2}{*}{$\begin{array}{l}\text { Ki-67 } \\
\text { index }\end{array}$} & \multirow[b]{2}{*}{$\mathrm{BM}$} & \multirow[b]{2}{*}{ LT } & \multirow[b]{2}{*}{ PET pattern } & \multicolumn{2}{|c|}{ Nodal FDG uptake } & \multirow{2}{*}{$\begin{array}{l}\text { Mediastinal } \\
\text { FDG uptake }\end{array}$} & \multirow{2}{*}{$\begin{array}{l}\text { Clinical } \\
\text { outcome }\end{array}$} \\
\hline & & & & & & & Intensity & SUVmax & & \\
\hline 1 & $\mathrm{M} / 33$ & T-LBL & 30 & + & + & Diffuse & Mild & 3.97 & + & $\mathrm{CR}(22 \mathrm{mo}+)$ \\
\hline 2 & $\mathrm{M} / 33$ & T-LBL & $\mathrm{X}$ & + & + & Diffuse & Intense & 11.1 & + & Early death \\
\hline 3 & $\mathrm{M} / 19$ & T-LBL & 60 & - & - & Localize & Intense & 12.2 & + & $\mathrm{CR}$ in death \\
\hline 4 & $\mathrm{M} / 40$ & T-LBL & 40 & + & - & Nodular & Intense & 8.37 & - & $\mathrm{CR}(30 \mathrm{mo}+)$ \\
\hline 5 & $\mathrm{~F} / 37$ & T-LBL & 50 & + & + & Diffuse & Moderate & 6.354 & + & PR in death \\
\hline 6 & $\mathrm{M} / 23$ & T-LBL & 60 & + & + & Diffuse & Mild & 3.72 & + & $\mathrm{CR}(16 \mathrm{mo}+)$ \\
\hline 7 & $\mathrm{M} / 29$ & T-LBL & 90 & + & + & Diffuse & Intense & 8.11 & + & $\mathrm{CR}(7 \mathrm{mo}+)$ \\
\hline 8 & $\mathrm{M} / 30$ & T-LBL & 70 & + & + & Diffuse & Mild & 4.58 & + & Early death \\
\hline 9 & $\mathrm{M} / 15$ & T-LBL & 30 & + & + & Diffuse & Mild & 4.2 & + & $\mathrm{CR}(7 \mathrm{mo}+)$ \\
\hline
\end{tabular}

T-LBL, T-lymphoblastic lymphoma; X, unknown; BM, bone marrow involvement; LT, leukemic transformation; CR, complete remission; PR, partial remission; CR/PR in death, succumbed to relapsed disease after experiencing CR or PR; mo+, time in months for latest follow-up.
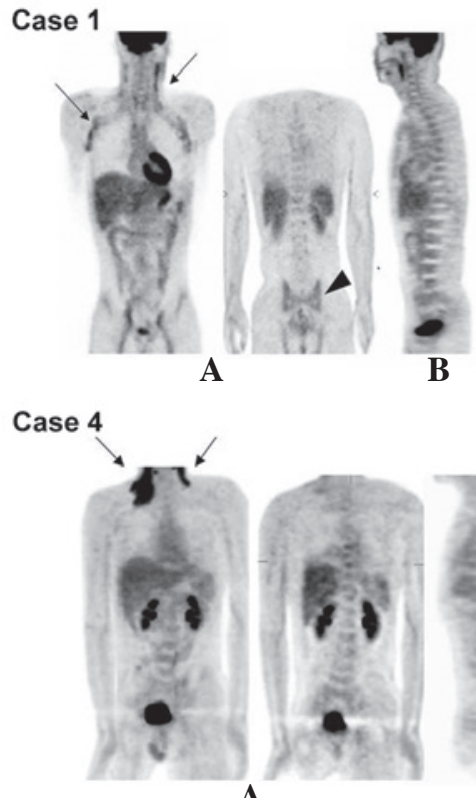

A

Case 7

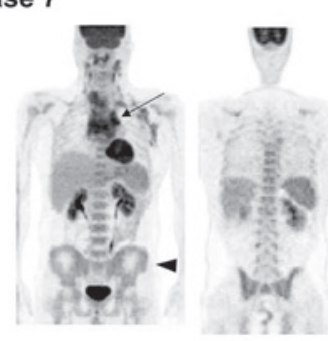

A

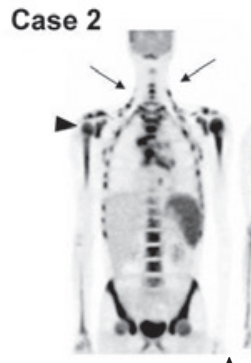

A

Case 5

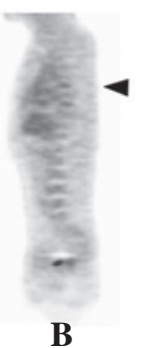

$\mathbf{B}$

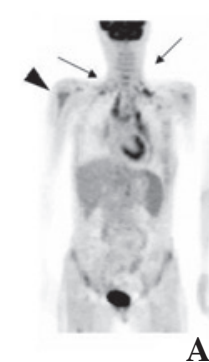

Case 8

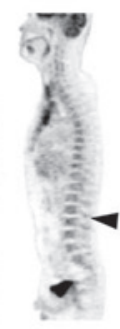

B
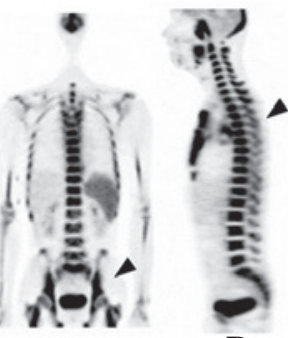

B

Case 3
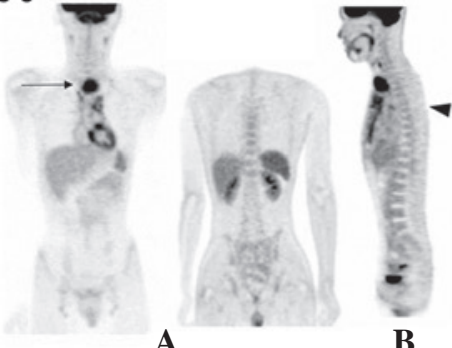

Case 6
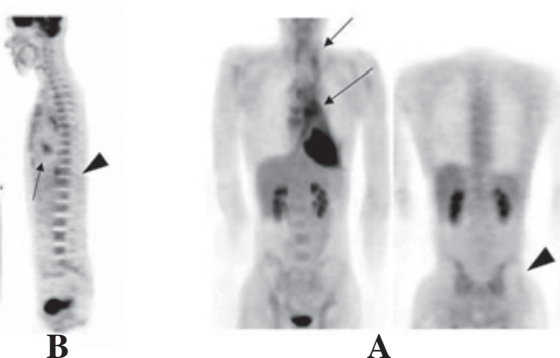

A

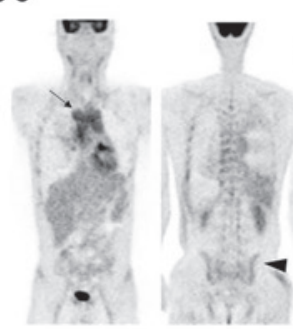

A

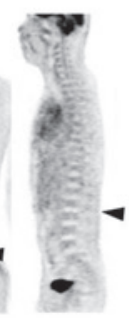

$\mathbf{B}$
Case 9

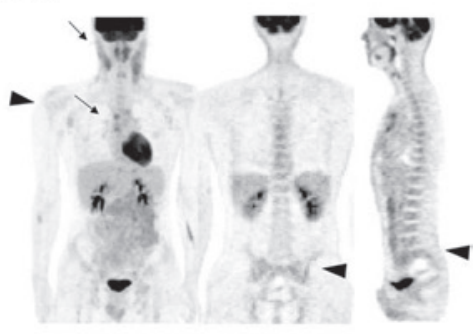

A

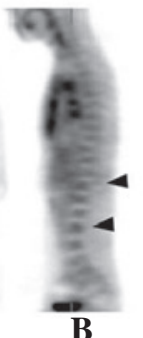

B

Figure 1. Fluorine-18 fluorodeoxyglucose positron emission tomography, (A) coronal and (B) sagittal images. Case 1: Confluent nodal activity is present in the bilateral neck and axilla (arrows), with diffuse and non-uniform BM activity (arrowhead). Case 2: Diffuse, hyperintense BM activity consistent with BM involvement. Activity is so extensive it is nearly confluent in areas (arrowhead). The bilateral jugular chains of the neck are shown (arrows). Case 3: Intense activity within the mediastinal mass (arrow). BM activity shows localized uptake (arrowhead). Case 4: Hyperintense activity within the neck and supraclavicular regions (arrows), with nodular BM activity at the level of the mid-T spines (arrowhead). Case 5: Nodal activities in the supraclavicular region, left axilla, mediastinum, bilateral pleura and pericardium (arrows), with diffuse and non-uniform BM activity (arrowhead). Case 6: Supraclavicular (arrow) and mediastinal leukemic transformation (arrows). BM shows heterogeneous hyperactivity, mainly in the thoraco-lumbar spine and hip (arrowhead). Case 7: Heterogneous nodal activity in the mediastinum (arrow) and supraclavicular area, with diffuse and non-uniform BM activity (arrowhead). Case 8: Triangular-shaped prevascular mediastinal activity (arrow) and diffuse BM hyperactivity (arrowhead). Case 9: Mild to moderate degree of hyperactivity in the neck, aortopulmonary window and subcarinal region (arrow). BM shows diffuse hyperactivity (arrowhead). BM, bone marrow. 
and fixed with formalin, and then cut into 2-mm thick sections. Bone marrow (BM) involvement or leukemic transformation (LT) was confirmed by iliac crest marrow biopsy and peripheral blood blast count.

FDG-PET image acquisition. FDG-PET scans were acquired at the initial pre-therapeutic period. Images were obtained with conventional PET scanners (GE Advance Scanner; GE Medical Systems, Waukesha, WI, USA; or ECAT EXACT HR+ Scanner; Siemens, Knoxville, TN, USA). All patients fasted for at least $6 \mathrm{~h}$ and serum glucose levels were $<180 \mathrm{mg} / \mathrm{dl}$ prior to scanning. At $60 \mathrm{~min}$ after the intravenous injection of 370-555 MBq FDG, the image acquisition was started.

Image analysis. Two experienced nuclear physicians assessed the FDG-PET images using visual analysis and maximum standardized uptakes (SUVmax). In the visual analysis, an increased tracer uptake pattern (diffuse, nodular or localized) and uptake intensity (mild, moderate or intense) were considered for assessment. The SUV was calculated as follows: $\mathrm{SUV}=$ mean activity [region of interest (ROI)] $(\mathrm{MBq} / \mathrm{ml})$ / injected dose $(\mathrm{MBq}) /$ total body weight $(\mathrm{g})$. Among these SUVs from the targeted ROI, the SUVmax were defined as the highest SUVs of pixels in the ROI.

\section{Results}

As shown in Table I, 8 patients presented with BM involvement; 7 showed LT and 1 patient showed BM involvement without LT at the initial diagnosis.

All lymphoma involvement lesions were FDG-avid and the intensity of nodal FDG uptake was variable (mild in 4, moderated in 1 and intense in 4 patient). The mean SUVmax was 6.4 \pm 3.3 (range, 3.7-12.2). As shown in Table I and Fig. 1, 8 out of 9 patients presented with hypermetabolic lesions in the mediastinum and intense splenic uptake was noted in 4 patients. A high Ki-67 index was associated with relatively intense and increased SUVmax values. All the images of the enrolled patients are presented in Fig. 1.

\section{Discussion}

The present study found two notable points in the FDG-PET imaging. First, all T-LBL patients with involvement of the BM showed diffuse or nodular BM FDG uptake. However, patients with no bone involvement showed localized marrow uptake (case 3; Fig. 1;). Second, with regard to LT, the LT patients presented with diffuse BM FDG uptake (mean SUVmax, 3.7 \pm 2.9 ), while the single patient with marrow involvement without LT showed nodular FDG uptake in the BM (SUVmax, 2.7) (case 4; Fig. 1). In the present study, all lymphomas involving lymph nodes and mediastinal lesions were FDG-avid. These results were concordant with previous studies (4,5). In addition, the present study provides a more detailed FDG uptake pattern with BM involvement and LT status. In conclusion, it is possible to use FDG-PET for the evaluation of the disease extent of T-LBL. Furthermore, it could provide a diagnostic clue for BM involvement or LT in T-LBL patients.

\section{References}

1. Hoelzer D, Gökbuget N, Digel W, Faak T, Kneba M, Reutzel R, Romejko-Jarosinska J, Zwolinski J and Walewski J: Outcome of adult patients with T-lymphoblastic lymphoma treated according to protocols for acute lymphoblastic leukemia. Blood 99: 4379-4385, 2002.

2. Ellin F, Jerkeman M, Hagberg $H$ and Relander T: Treatment outcome in T-cell lymphoblastic lymphoma in adults - a population-based study from the Swedish Lymphoma Registry. Acta Oncol 53: 927-934, 2014.

3. Seam P, Juweid ME and Cheson BD: The role of FDG PET scans in patients with lymphoma. Blood 110: 3507-3516, 2007.

4. Weiler-Sagie M, Bushelev O, Epelbaum R, Dann EJ, Haim N, Avivi I, Ben-Barak A, Ben-Arie Y, Bar-Shalom R and Israel O: (18)F-FDG Avidity in lymphoma readdressed: A study of 766 patients. J Nucl Med 51: 25-30, 2010.

5. Xiang X, Wang X, Yi Q, Lin L, Zhang X, Liang H, Yang J: Precursor T-cell lymphoblastic lymphoma extensively involving the mediastinum, pleura and pericardium: A case report. Mol Clin Oncol 2: 945-948, 2014. 